\title{
Sedimentmanagement an alpinen Speichern Das EU-INTERREG IIIB Projekt ALPRESERV
}

\author{
Sediment Management in Alpine Reservoirs \\ The ALPRESERV EU INTERREG IIIB Project
}

\author{
von H. KNOBLAUCH, S. HARTMANN und G. DE CESARE
}

\section{KURZFASSUNG/SUMMARY}

Die Speicher wasserbaulicher Anlagen im Alpenraum erfüllen vielfache Zwecke. So dienen sie zur Erzeugung elektrischer Energie, zur Sicherung des Hochwasserschutzes und zur Gewinnung von Trinkwasser. Die Nachhaltigkeit der Speicher kann jedoch durch die Verlandung infolge des Sedimenttransportes beeinträchtigt werden. Dies gilt sowohl für hochalpine Speicher wie auch für Speicher an Flüssen. Demgegenüber können die fehlenden Feststoffe die ökologische Vielfalt, die Stabilität der Flusssohle und somit auch den Grundwasserstand im Unterlauf der Absperrbauwerke beeinflussen. Aus diesen Gründen sind die Betreiber von wasserbaulichen Anlagen bemüht, ein nachhaltiges Sedimentmanagement vorzusehen. Das EU INTERREG IIIB Projekt ALPRESERV wurde initiiert, um auf einer internationalen Ebene diese Bemühungen in Bezug auf die wasserwirtschaftlichen, rechtlichen und ökologischen Randbedingungen zusammenzufassen und einen gegenseitigen Erfahrungsaustausch zu ermöglichen.

The reservoirs of hydro-electric developments in the Alps serve different purposes: power generation, flood protection, drinking-water supply. The sustainability of such reservoirs may, however, be jeopardised by their filling up with sediments. This is true of both high-head schemes in the Alps and low-head schemes on rivers. On the other hand, the lack of solids in streamflow risks affecting the ecological diversity, riverbed stability, and the water table downstream of the dams. For these reasons, the operators of hydro-electric plant attempt to provide for sustainable sediment management. The ALPRESERV EU INTERREG IIIB project has been launched to pool efforts relating to water resources management, law, and ecology on an international level and to enable the mutual exchange of experience.

\section{SEDIMENTMANAGEMENT IM ALPENRAUM}

Die alpinen Regionen bilden aufgrund ihres morphologischen Charakters und klimatologischen Gefüges eine vielfältige Landschaft. Aus diesen Gründen ist ein einheitlich prognostizierbares Modell eines Feststoffverhaltens in Einzugsgebieten von Bächen und Flüssen nicht zu formulieren. In Bezug auf die Transportprozesse von Feststoffen stellen die jeweiligen hydrologischen, geomorphologischen und klimatischen Randbedingungen die wesentlichsten Einflussfaktoren dar. Darüber hinaus müssen die räumlichen und zeitlichen Veränderungen bei der Abschätzung und Bewertung von Transportraten berücksichtigt werden. Die Alpen selbst zählen zusammen mit dem Himalaya-Gebirge, den Anden und den Gebirgszügen an der pazifischen Küste Amerikas mit zu den Gebieten mit der höchsten Erosionsrate. Erosionsraten von mehr als $1000 \mathrm{t} / \mathrm{km}^{2} \cdot \mathrm{a}$ können hier auftreten (WALLING und WEBB, 1983, siehe Abb. 1). Spitzenreiter in dieser Aufstel-

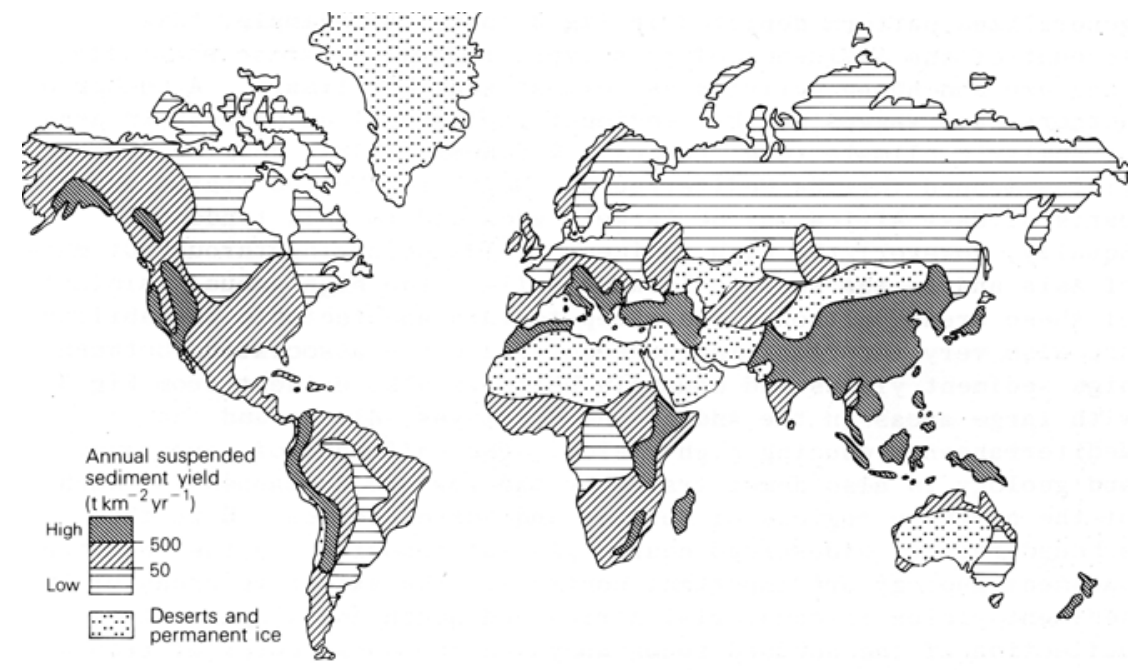

lung ist das Gebiet zwischen Australien und Asien. Hier werden Erosionsraten zwischen 10000 bis $50000 \mathrm{t} / \mathrm{km}^{2} \cdot \mathrm{a}$ gemessen (WALLING, 1994).

Neben dem Niederschlag stellen die geomorphologischen Effekte, verursacht durch Gletscher, Lawinen, Muren, Bodenerosionen etc., den größten Einfluss auf die Erosionsrate und somit auf den Feststofftransport in Bächen und Flüssen dar. Dieser wird sehr stark, auch jahreszeitlich bedingt, von der Vegetation und der Versiegelungsrate geprägt. Weitere Einflussfaktoren auf die oben erwähnten Prozesse stellen vulkanische und tektonische Aktivitäten sowie anthropogene Einflüsse dar. Besonders letztere können sich stark steigende Erosionsraten bei einer Änderung der Bodenbedeckung verursachen.

Durch fluviale Prozesse werden die erodierten Feststoffe weiter transportiert, ehe sie sich bei entsprechenden Randbedingungen in natürlichen bzw. künstlichen Seen und Speichern absetzen. Die Reduktion des Stauraumes, das Verlegen der Betriebseinrichtungen, eine Reduktion der Hochwassersicherheit, die Verlegung des Schotterlückenraumes, fehlender Feststoff im Unterlauf, die Beeinträchtigung von Freizeiteinrichtungen etc. können die negative Konsequenz sein. Maßnah- 
men zur Bewältigung dieser Auswirkungen zielen daher vor allem darauf $\mathrm{ab}$,

- den Eintrag an Feststoffen in den Speicher zu minimieren,

- die Verlandungsrate im Speicher selbst zu minimieren bzw.

- die angelandeten Sedimente aus dem Speicher zu entfernen.

Die anzuwendende Maßnahme hängt von der Art und dem Verwendungszweck sowie vom ökologischen und gesellschaftlichen Umfeld der Anlage ab. Allgemeine Grundsätze zur Bewältigung des Problems können zwar angegeben werden, zur Auswahl einer Maßnahme sind jedoch immer die spezifischen Randbedingungen der jeweiligen Anlage zu berücksichtigen.

\section{DAS EU-INTERREG IIIB PROJEKT ALPRESERV}

\section{1 Überblick}

Im Rahmen des EU-initiierten INTERREG IIIB Programms wurde das Projekt ALPRESERV gestartet, das 17 ProjektpartnerInnen aus 5 Ländern (Deutschland, Österreich, Italien, Schweiz und Slowenien) vereint. Die Ziele des Projekts bestehen darin, durch einen Wissensaustausch, durch
Pilotprojekte und eine gemeinsame Datenbank die Probleme der Verlandung von Speichern im alpinen Raum auf einer internationalen ExpertInnenebene zu diskutieren und gemeinsam Lösungen zu erarbeiten.

Das durch Mittel der EU (European Regional Development Fund ERDF) geförderte INTERREG III Programm zielt daraufhin ab, die ökonomische und soziale zwischenstaatliche und überregionale Kooperation und Kommunikation zu fördern.

Das Subprogramm ALPINE SPACE (http://www.alpinespace.org/) bezieht sich räumlich gesehen auf die Alpen-Anrainerstaaten (siehe Abb. 2) mit den vier Schwerpunktthemen der Entwicklung einer nachhaltigen Raumordnung, der Förderung zwischenstaatlicher Projekte, der Entwicklung effizienter Lösungen für Transport- und Kommunikationsprobleme und dem Schutz der Bevölkerung sowie der Infrastruktur vor den Auswirkungen natürlicher Katastrophen.

ALPRESERV („Sustainable Sediment Management of ALPine RESERVoirs considering ecological and economical aspects“) zählt im Rahmen des INTERREG Programms zum Kapitel 3, das Maßnahmen in der Natur

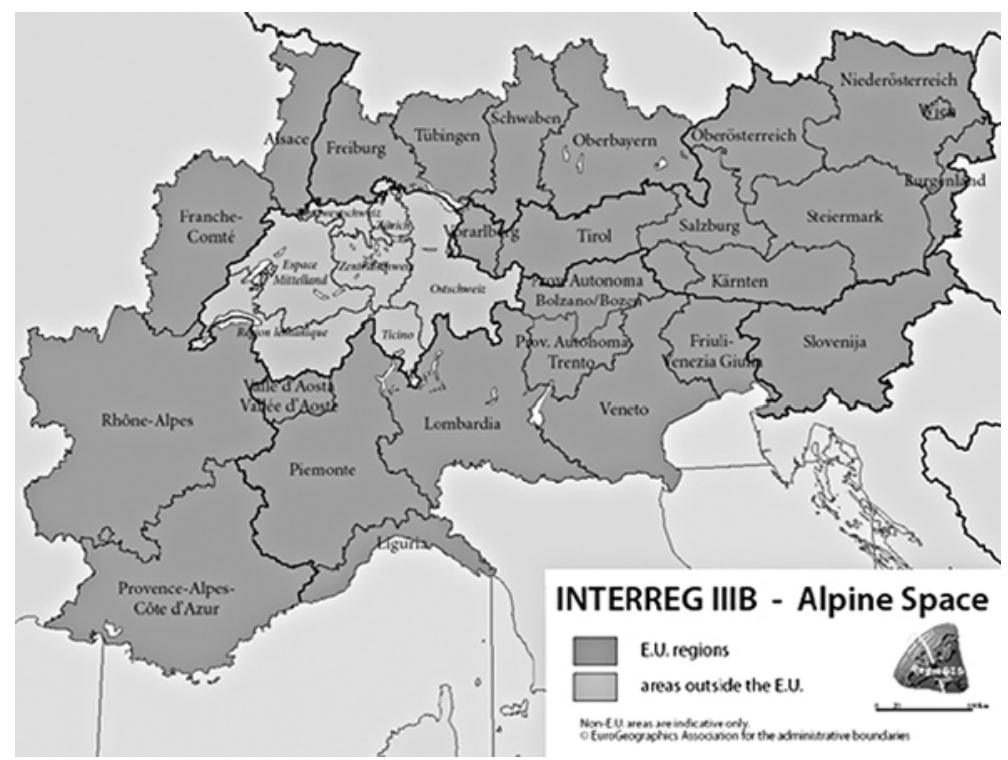

Abb. 2. Die räumliche Erstreckung des INTERREG IIIB-Alpine Space Programmes
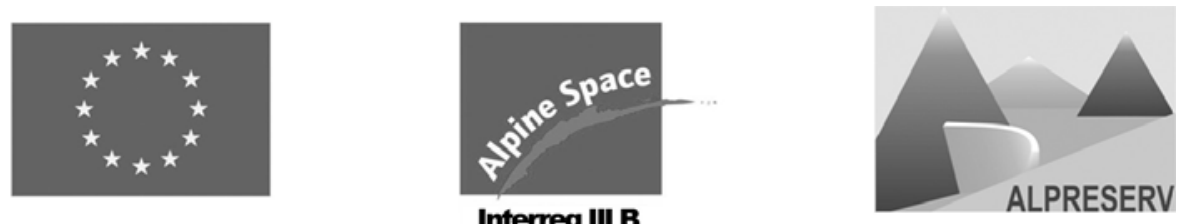

Abb. 3. Symbole des EU-INTERREG IIIB Projektes ALPRESERV und Ressourcen, im Speziellen das Wasser betreffend, zum Inhalt hat. Basierend auf der großen Bedeutung des Wassers für den Alpenraum als natürliche Ressource für Trinkwasser und Erzeugung elektrischer Energie soll das Problem der Speicherverlandung umfassend behandelt werden. Betriebliche Beeinträchtigungen wie auch ökologische Auswirkungen werden theoretisch erfasst wie auch anhand von $7 \mathrm{Pi}$ lotprojekten praktisch behandelt. Die Randbedingungen für einen Maßnahmenkatalog werden aus technischer, rechtlicher und ökologischer Sicht zusammengefasst und sollen über eine Datenbank interessierten Personen wie auch Institutionen zur Verfügung gestellt werden. Die Laufzeit des Projekts erstreckt sich von April 2003 bis Ende 2006.

\subsection{Die Projektpartnerlnnen}

Das Projekt ALPRESERV vereint 17 ProjektpartnerInnen aus 5 Ländern unter der Leitung der Universität der Bundeswehr München.

Die ProjektpartnerInnen aus Deutschland:

- Universität der Bundeswehr München

- Bayerisches Staatsministerium für Umwelt, Gesundheit und Verbraucherschutz, München

- Schneider \& Jorde Ecological Engineering $\mathrm{GmbH}$, Stuttgart

Die ProjektpartnerInnen aus Italien:

- Universitá degli Studi di Trieste, Dipartimento di Ingegneria Civile

- Autonome Provinz Bozen-Südtirol, Ämter für Jagd und Fischerei, Stauanlagen und Wasserwirtschaft

- CESI, Bergamo

- Provincia di Belluno, Settore Lavori Pubblici, Difesa del Suolo e Tutela Ambiente

Die ProjektpartnerInnen aus der Schweiz:

- Ecole Polytechnique Fédérale Lausanne, Laboratoire de constructions hydrauliques

- Kraftwerke Gougra AG

- Dienststelle für Wasserkraft des Kanton Wallis

- Schweizerischer Wasserwirtschaftsverband

Der ProjektpartnerIn aus Slowenien:

- University of Ljubljana, Hydrotechnical Department

Die ProjektpartnerInnen aus Österreich: 

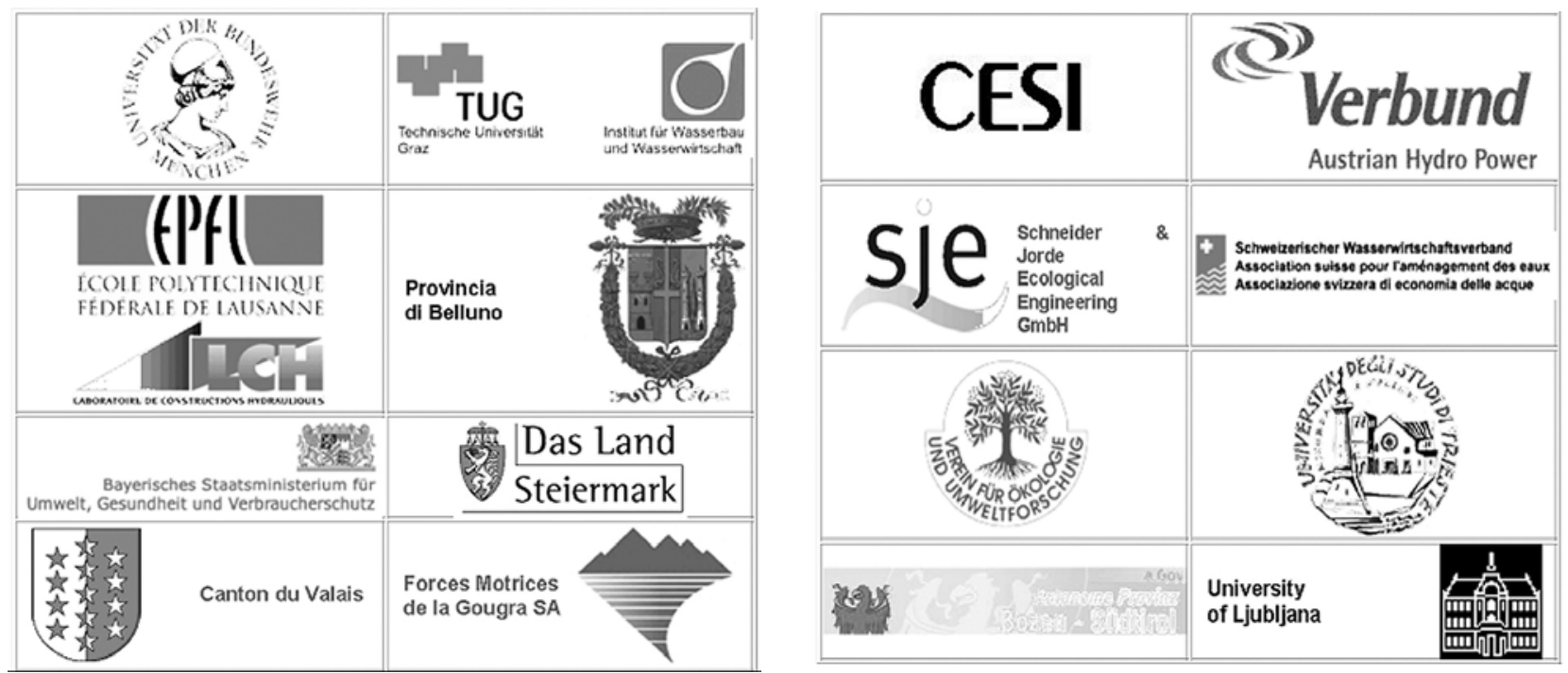

Abb. 4. Die Projektpartner von ALPRESERV

- Technische Universität Graz, Institut für Wasserbau und Wasserwirtschaft

- Amt der Steiermärkischen Landesregierung, Fachabteilung 19A, Wasserwirtschaftliche Planung und Hydrographie

- Amt der Steiermärkischen Landesregierung, Fachabteilung 13A, Umweltrecht und Energie

- Österreichischer Verein für Ökologie und Umweltforschung

- Verbund - Austrian Hydro Power AG (AHP)

\subsection{Die Arbeitsschwerpunkte von ALPRESERV}

Das Projekt gliedert sich in 9 Bereiche („Work Packages“) mit folgenden Inhalten:

- Work Package 1: Zwischenstaatliche Projektvorbereitung. Das bereits bestehende Netzwerk zwischen den 4 Universitäten wurde durch öffentliche Verwaltungsstellen, einschlägige Firmen sowie Vereine erweitert und das Konsortium somit fachlich bereichert und ergänzt. In diesem Bereich sind des Weiteren die organisatorischen Kontakte zwischen den einzelnen ProjektpartnerInnen und der verantwortlichen Behörde und die formalen Abwicklungen sowie die Organisation regelmäßiger Konferenzen integriert.

- Work Package 2 und 3: Projektmanagement. In Erweiterung des Work Package 1 wurden hier die Kooperationen und Kommunikationen zwischen dem Koordinator und den ProjektpartnerInnen be- handelt, die einen wesentlichen Teil des Projektes bilden.

- Work Package 4: Öffentlichkeitsarbeit. Über mehrere Schienen werden die Ergebnisse des Projekts veröffentlicht und präsentiert. So informiert eine Homepage (http:// www.ALPRESERV.org/) über den aktuellen Projektsstand. Eine Publikationsserie, die die einzelnen Schwerpunkte erläutern soll, ist gerade in Vorbereitung und ein Symposium zum Thema der Stauraumverlandung am Ende des Projekts wird die wesentlichen Ergebnisse präsentieren.

- Work Package 5: Sedimentquellen und Transportprozesse. In einem eigenen Bericht werden die Feststoffherde im alpinen Bereich und die den Transport kennzeichnenden Parameter beschrieben. Das Ziel ist die Identifizierung der für den jeweiligen Speicher kennzeichnenden Parameter für den Eintrag an Feststoffen.

- Work Package 6: Sedimentation in Speichern. Auf der Grundlage des technischen Kenntnisstandes werden die Prozesse der Stauraumverlandung aufgezeigt und Möglichkeiten der Behandlung erläutert. Einen besonderen Schwerpunkt bilden hier die Dichteströmungen mit den Möglichkeiten, Präventivmaßnahmen wie Einbauten, die Anordnung von Luftschleiern, Wasserstrahlen anzusetzen.

- Work Package 7: Maßnahmen für ein nachhaltiges Sedimentmanagement. Behandelt werden die drei hauptsächlichen Strategien zur Be- handlung des Problems der Stauraumverlandung: die Minimierung der Sedimentfrachten, die in den Speicher gelangen; die Minimierung der Sedimentation; das Entfernen bereits abgelagerten Materials. Einen weiteren Schwerpunkt bildet das Thema des fehlenden Geschiebes unterhalb einer Stauanlage.

- Work Package 8: Pilotprojekte (siehe auch Kapitel 3). An sieben ausgewählten Stauanlagen in Österreich, Deutschland, Schweiz und Italien werden beispielhaft Maßnahmen mit der Zielsetzung eines nachhaltigen Sedimentmanagements durchgeführt. Die Anlagen wurden ausgewählt, um einen möglichst breiten Bereich von Problemstellungen zum Thema der Stauraumverlandung zur Verfügung zu haben. Die Ergebnisse der Maßnahmen wurden und werden im Zuge der regelmäßigen Besprechungen den ProjektspartnerInnen zur Kenntnis gebracht und anschließend in die Datenbank integriert. Diese Datenbank soll in $\mathrm{Zu}$ kunft als Informationsquelle für interessierte BetreiberInnen und Behörden dienen, um ähnlich gelagerte Fälle zielgerichteter behandeln zu können. Gedacht ist an eine Datenbank, die über das Internet öffentlich zugängliche Informationen zu den Anlagen, den Problemstellungen und den gefundenen Lösungen bietet.

- Work Package 9: Analyse der Auswirkungen. Ein Monitoring Programm wird die Auswirkungen der Maßnahmen bei den Pilotprojek- 


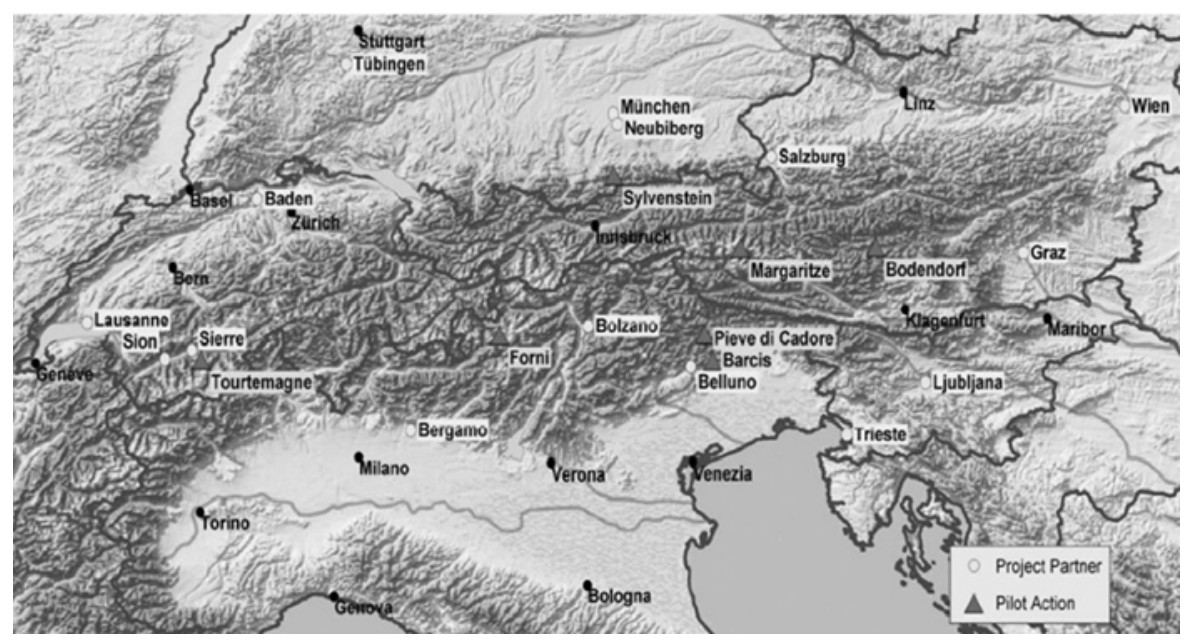

Abb. 5. Lokalisierung der Pilotprojekte (Tourtemagne, Formi, Barcis, Pieve di Cadore, Sylvenstein, Margaritze, Bodendorf) und der Projektpartner (Lausanne, Baden, Sion, Sierre, Bergamo, Bolzano, Belluno, Trieste, Ljubljana, Tübingen, Neubiberg, München, Salzburg, Wien, Graz)

ten protokollieren. Dies betrifft die jeweilige Feststoffbilanz wie auch die ökologische Situation des Flussgebietes.

\section{DIE PILOTPROJEKTE}

Die Auswahl der sieben Pilotprojekte erfolgte unter dem Kriterium, ein möglichst breites Spektrum von Problemstellungen zum Thema der Stauraumverlandung zur Verfügung zu haben. Berücksichtigt wurden ein Flusskraftwerk, Speicher im voralpinen Bereich und weitere in der alpinen Zone.

\subsection{Kraftwerk Bodendorf}

In diesem Fall handelt es ich um ein Flusskraftwerk, das als Kopfspeicher im Oberlauf der Mur situiert ist. Es befindet sich im Eigentum der VerbundAustrian Hydro Power AG. Oberhalb des Stauraumes befindet sich eine freie Fließstrecke, in der das Flusssediment ungehindert transportiert werden kann. Größtenteils lagert sich das transportierte Sediment im Stauraum Bodendorf ab. Daraus ergeben sich Anlandungen, die im Bereich der Stauwurzel zum Anheben der Wasserspiegel führen. Weiters kann angelandetes Geschiebe zu Funktionsbeeinträchtigung der Betriebsorgane (Krafthaus und Wehranlage) führen. Seit dem Jahre 1994 fanden bislang fünf Stauraumentlandungen statt.

Im Bereich der Stauwurzel des KW Bodendorf wurden im Oktober 2003 Kurzbuhnen und eine Initialrinne errichtet. Die Maßnahmen sollen als Ertüchtigung des Geschiebetransports und somit zum verbesserten Abtrag der Anlandungen im Stauwurzelbereich führen. Im Rahmen des Projekts
ALPRESERV wurde ein Monitoring für die Stauraumentlandung im Jahr 2004 durchgeführt, um die Auswirkungen der Buhnen und der Initialrinne $\mathrm{zu}$ evaluieren und eine $\mathrm{Ge}$ schiebe- und Schwebstoffmassenbilanz für die Entlandung $2004 \mathrm{zu}$ erstellen.

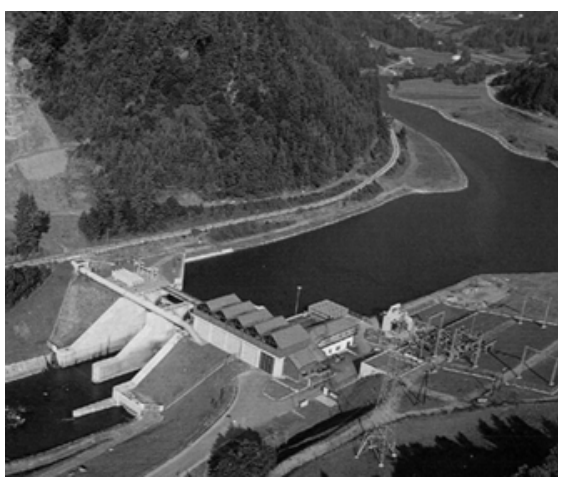

Abb. 6. Das KW Bodendorf (Quelle: Verbund AHP)

\subsection{Speicher Margaritze}

Der Hochgebirgsspeicher Margaritze ist Teil der Kraftwerksanlage GlocknerKaprun und befindet sich ebenfalls im Eigentum der Verbund-Austrian Hyddro Power AG. Er trägt wesentlich zur Spitzenenergieerzeugung der Kraftwerksgruppe bei. Die Abb. 5 zeigt ein Bild des Speichers mit den beiden Sperrenbauwerken Möllsperre und Margaritzensperre. Dem Speicher vorgelagert ist ein natürlicher Gletschersee, der bis in die 90er Jahre Sediment zurückgehalten hat. Durch die komplette Verlandung des Gletschersees wird nun das Sediment im Ausmaß von im Durchschnitt $40000 \mathrm{~m}^{3} / \mathrm{a}$ im Speicher Margaritze abgelagert.
Aus diversen Gründen ist es nicht möglich, eine Speicherspülung durchzuführen. Derzeit wird daher das Sediment mittels einer Baggerpumpe vom Schluchtteil in den Flachteil umgelagert, um den Bereich der Grundablässe frei von Sediment zu halten. Zum Teil fließt das umgelagerte Sediment vom Flachteil zurück in den Schluchtteil der Stauhaltung. Im Zuge des gegenständlichen EU-Projektes werden die Strömungsverhältnisse für verschiedene Randbedingungen untersucht. Dabei kommen in erster Linie Messgeräte zum Einsatz, die mittels Ultraschall die Strömungsgeschwindigkeiten dreidimensional erfassen. Zusätzlich werden Trübemessungen durchgeführt und Schwebstoffproben gesammelt, analysiert und ausgewertet.

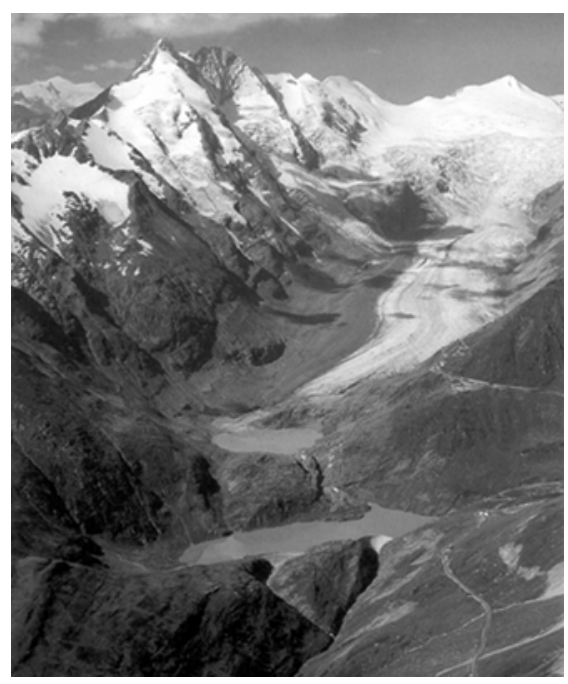

Abb. 7. Speicher Margaritze (Vordergrund), Sandersee (Hintergrund) und Großglockner (Quelle: Verbund - AHP)

\subsection{Speicher Sylvenstein}

Diese Mehrzweckanlage liegt im Oberlauf der Isar in Oberbayern. Durch den Bau des Dammes wurde der Sedimenttransport beinahe gänzlich unterbrochen, der Speicher verlandet. Vordämme in den Zulaufbereichen schützen die Betriebseinrichtungen vor der Verlandung. Im Rahmen des ALPRESERV-Projekts sollen die Fragen nach der chemischen und physikalischen Zusammensetzung des Sediments, der mögliche Verwendungszweck, die mögliche Minimierung des Sedimentzulaufs und die beste Möglichkeit einer Entfernung der Feststoffe aus dem Speicher geklärt werden.

\subsection{Speicher Tourtemagne}

Die Wasserkraftanlage liegt im Wallis, Schweiz. Durch den Rückgang des 


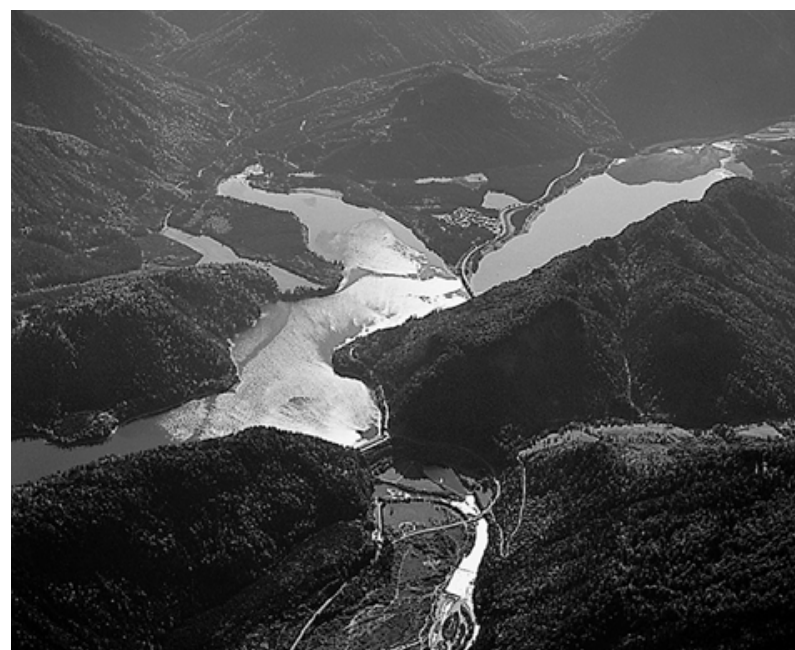

Abb. 8. Ansicht Speicher Sylvenstein mit dem Abschlussbauwerk (Quelle: Wasserwirtschaftsamt Weilheim)

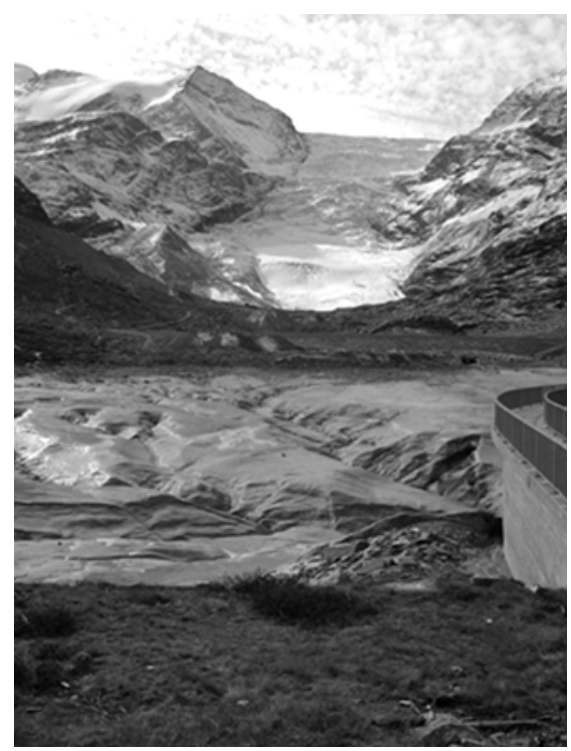

Abb. 9. Absperrbauwerk der Anlage Tourtemagne mit dem verlandeten Speicher

halb des Speichers wurde bereits ein Entsander gebaut, um den weiteren Eintrag an Feststoffen weitgehend
Gletschers wurde der Bau der Anlage im Jahre 1960 ermöglicht. Der weitere Rückzug des Gletschers resultierte in der Verlandung des Speichers. Ober zu zu verhindern. Die bereits abgesetzten Feststoffe wurden bislang teilweise mechanisch aus dem Stauraum entfernt. Regelmäßige Spülungen ergänzen die Maßnahmen des Sedimentmanagements. Zukünftig soll ein dynamisches Sedimentmanagement, das sich auf das gesamte Einzugsgebiet erstreckt, angewandt werden, um den Sedimenteintrag in den Speicher zu minimieren. Dazu zählen unter anderem künstliche Hochwässer in Verbindung mit Spülungen wie auch die Konstruktion eines Umleitungsstollens.

\subsection{Speicher Barcis}

Die Anlage liegt in Friaul, Oberitalien. Der hohe Sedimenteintrag reduzierte das nutzbare Stauvolumen um ca. 1/3. Als Maßnahme wurde bislang ein kleiner Vordamm errichtet, dessen Rück- stauraum sich jedoch bereits wieder sehr schnell gefüllt hat. Wegen der touristischen Nutzung des Gebietes ist die mechanische Räumung und Abtransport auf Straßen nur bedingt möglich. Im Rahmen des ALPRESERV-Projekts sollen daher neue Strategien erforscht und angewandt werden.

\subsection{Speicher Forni}

Die Anlage liegt in der Lombardei, westliches Oberitalien. Der stark sedimenthaltige Zufluss, dessen Einzugsgebiet vergletschert ist, hat das nutzbare Speichervolumen bereits stark reduziert. Als Maßnahme wird derzeit beinahe zweimal jährlich eine Spülung des Speichers durchgeführt. Die ausgespülten Sedimente drohen nun die Hochwassersicherheit im Unterlauf zu gefährden.

\subsection{Speicher Pieve di Cadore}

Die Anlage liegt im Piave-Tal nördlich von Belluno, Oberitalien. Die Mehrzweckanlage mit dem $6 \mathrm{~km}$ langen Speicher dient der Stromerzeugung, der Bewässerung und dem Hochwasserschutz. Der hohe Feststoffanteil des Flusses hat zu einer bedeutenden Reduktion des Speichervolumens geführt. Im Rahmen des Projekts ALPRESERV sollen die bisher bereits durchgeführten Untersuchungen bezüglich der Korngrößenverteilung, der mineralischen und chemischen $\mathrm{Zu}$ sammensetzung der Sedimente in Bezug auf die Ablagerungshöhe im Speicher ausgeweitet und ergänzt werden.

\section{DATENBANK}

Parallel zu den Arbeiten und Untersuchungen an den Pilotprojekten wird eine Datenbank aufgebaut. Basierend auf den charakteristischen Informationen zu den jeweiligen Anlagen wird diese Datenbank speziell auf

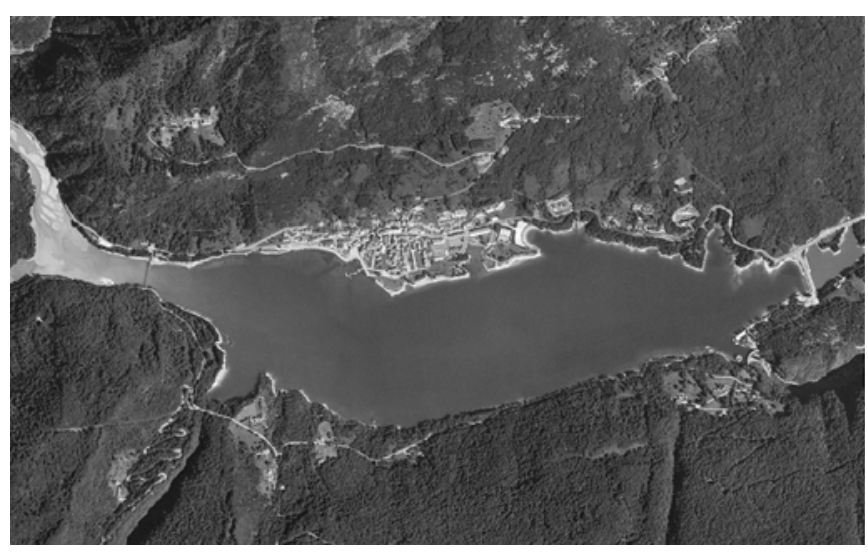

Abb. 10. Ansicht Speicher Barcis

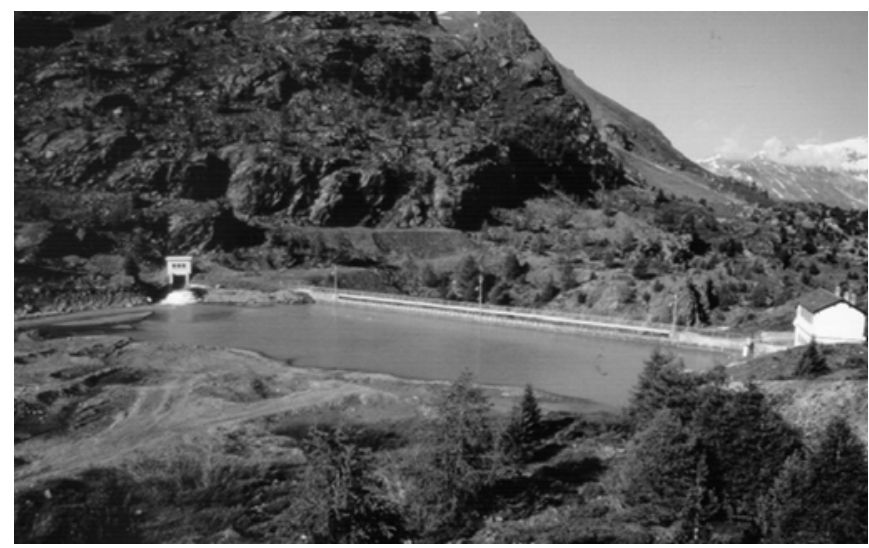

Abb. 11. Der Speicher Forni 


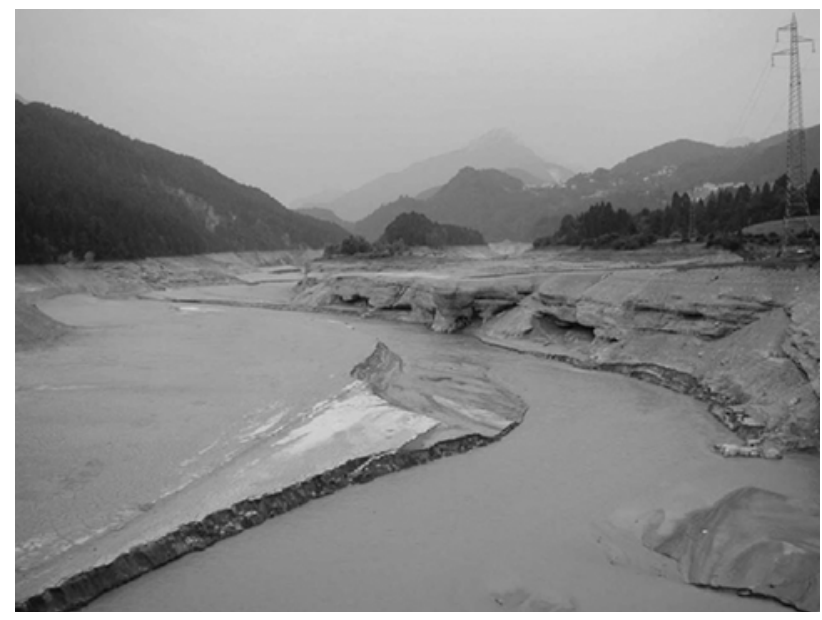

Abb. 12. Anlandungen im abgesenkten Speicher Pieve di Cadore (Quelle: Provincia di Belluno)

die Belange der Stauraumverlandung ausgerichtet. Dadurch wird die jeweilige Anlage in Bezug auf ihre Sedimentproblematik katalogisiert und in ein bestimmtes Ordnungsschema eingereiht. Das Ziel ist der (internationale) Informationsaustausch, wobei bereits gemachte Erfahrungen leichter nachvollziehbar sein sollen und weiteren Anwendungen zur Verfügung stehen können. Den Beginn werden die Ergebnisse der Pilotprojekte darstellen. Der Bereich der AnwenderInnen erstreckt sich auf die BetreiberInnen der Anlagen, Behörden, ÖkologInnen, PlanerInnen, Maschinenherstel-
lerInnen, Gemeinden, örtliche Vertretungen etc.

\section{ZUSAMMENFASSUNG}

Die Möglichkeiten, Maßnahmen gegen Stauraumverlandungen zu setzen, sind begrenzt und werden zusätzlich durch wirtschaftliche, technische bzw. durch rechtliche Aspekte beschränkt. Das EU-INTERREG IIIB Projekt ALPRESERV soll auf der Grundlage von Betriebserfahrungen die nachhaltige Bewirtschaftung von Speichern ermöglichen. Das Ziel der nachhaltigen Speicherbewirtschaftung ist der Erhalt der Funktionsfähigkeit der Anlagen durch entsprechende Maßnahmen.

Dazu sollen unter Berücksichtigung nationaler Richtlinien und der EU-Wasserrahmenrichtlinie internationale Erfahrungen in eine Datenbank einfließen und öffentlich zur Verfügung gestellt werden. Pilotprojekte an sieben Speichern im Alpenraum dienen als Referenzen, an denen exemplarisch Maßnahmen in Bezug auf die Entlandung eines Speichers durchgeführt werden. 0
WALLING, D. E., WEBB, B. E. (1983): Patterns of sediment yield. In: GREGORY, K.J. (ed.), Background to palaeo-hydrology. John Wiley, New York, 69-100.

WALLING, D. E. (1994): Measuring sediment yields from river basins. In: LAB, R. (ed.), Soil erosion research methods. Soil and Water Conservation Society, Ankeny, lowa.

Anschrift der Verfasser: Dipl.-Ing. Dr. Helmut KNOBLAUCH, Universitätsassistent am Institut für Wasserbau und Wasserwirtschaft, Techni-

\section{LITERATUR}

sche Universität Graz, Stremayrgasse 10, 8010 Graz, Österreich; E-Mail: helmut.knoblauch@tugraz.at; Dr. Ing. Sven HARTMANN, Universität der Bundeswehr München, Institut für Wasserwesen, Werner-Heisenberg-Weg 39, 85577 Neubiberg, Deutschland; sven.hartmann@unibwmuenchen.de; Dr. Giovanni DE CESARE, Laboratoire de Constructions Hydrauliques (LCH), Ecole Polytechnique Fédérale de Lausanne (EPFL), GC A3 484 (Bâtiment GC), Station 18, 1015 Lausanne, Schweiz; giovanni.decesare@epfl.ch. 